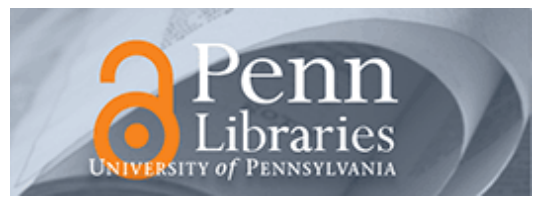

University of Pennsylvania

ScholarlyCommons

Statistics Papers

Wharton Faculty Research

2008

\title{
A Bivariate Timing Model of Customer Acquisition and Retention
}

David A. Schweidel

Peter S. Fader

University of Pennsylvania

Eric T. Bradlow

University of Pennsylvania

Follow this and additional works at: https://repository.upenn.edu/statistics_papers

Part of the Applied Statistics Commons, Business Administration, Management, and Operations

Commons, Business Analytics Commons, Business and Corporate Communications Commons, Management Information Systems Commons, Management Sciences and Quantitative Methods Commons, and the Statistical Models Commons

\section{Recommended Citation}

Schweidel, D. A., Fader, P. S., \& Bradlow, E. T. (2008). A Bivariate Timing Model of Customer Acquisition and Retention. Marekting Science, 27 (5), 829-843. http://dx.doi.org/10.1287/mksc.1070.0328

This paper is posted at ScholarlyCommons. https://repository.upenn.edu/statistics_papers/660

For more information, please contact repository@pobox.upenn.edu. 


\title{
A Bivariate Timing Model of Customer Acquisition and Retention
}

\begin{abstract}
Two widely recognized components, central to the calculation of customer value, are acquisition and retention propensities. However, while extant research has incorporated such components into different types of models, limited work has investigated the kinds of associations that may exist between them. In this research, we focus on the relationship between a prospective customer's time until acquisition of a particular service and the subsequent duration for which he retains it, and examine the implications of this relationship on the value of prospects and customers.
\end{abstract}

To accomplish these tasks, we use a bivariate timing model to capture the relationship between acquisition and retention. Using a split-hazard model, we link the acquisition and retention processes in two distinct yet complementary ways. First, we use the Sarmonov family of bivariate distributions to allow for correlations in the observed acquisition and retention times within a customer, next, we allow for differences across customers using latent classes for the parameters that govern the two processes. We then demonstrate how the proposed methodology can be used to calculate the discounted expected value of a subscription based on the time of acquisition, and discuss possible applications of the modeling framework to problems such as customer targeting and resource allocation.

\section{Keywords}

customer acquisition, customer retention, customer retention management, stochastic models

\section{Disciplines}

Applied Statistics | Business | Business Administration, Management, and Operations | Business Analytics | Business and Corporate Communications | Management Information Systems | Management Sciences and Quantitative Methods | Statistical Models | Statistics and Probability 


\title{
A Bivariate Timing Model of
}

\author{
Customer Acquisition and Retention
}

\section{David A. Schweidel ${ }^{*}$}

Peter S. Fader

Eric T. Bradlow

*David A. Schweidel is a doctoral candidate, Peter S. Fader is the Frances and Pei-Yuan Chia Professor,
Professor of Marketing, and Eric T. Bradlow is K.P. Chao Professor, Professor of Marketing and Statistics
and Academic Director of the Wharton Small Business Development Center, The Wharton School of the
University of Pennsylvania. The authors express their appreciation to a requested anonymous firm that
graciously provided the data used here. Please address all correspondence on this manuscript to David
Schweidel, dschweid@wharton.upenn.edu, 3730 Walnut Street, Suite 700 JMHH, Philadelphia, PA 19104. 


\title{
A Bivariate Timing Model of \\ Customer Acquisition and Retention
}

\begin{abstract}
$\underline{\text { Abstract }}$
Two components central to the calculation of customer value are acquisition and retention propensities. However, while extant research has incorporated such components into different kinds of models, limited work has investigated the kinds of associations that may exist between them. In this research, we focus on the relationship between a prospective customer's time until acquisition of a particular service and the subsequent duration for which he retains it, and examine the implications of this relationship on the (lifetime) value of customers.

To accomplish these tasks, we utilize a bivariate timing model to capture the relationship between acquisition and retention. We incorporate duration dependence into each of the two marginal timing processes, and link them in two ways. First, we use the Sarmonov family of bivariate distributions to allow for correlations in the observed acquisition and retention times; next we allow for latent classes in the parameters that govern the two processes. We then show how the proposed methodology can be used to calculate the discounted expected tenure of service based on the time of acquisition, and discuss possible applications of the modeling framework to problems such as customer targeting and resource allocation.
\end{abstract}

Keywords: Customer Acquisition, Customer Retention, Customer Relationship Management, Stochastic Models 


\section{Introduction}

Service acquisition and retention have been tied to managerial metrics such as the value of the customer base (e.g., Gupta, Lehmann, and Stuart 2004) and managerial decisions such as resource allocation (e.g., Blattberg and Deighton 1996). These two constructs - the time that elapses before a prospective customer acquires a particular service and the subsequent duration for which a customer retains service after acquiring it - may be related to each other. Yet only a limited number of papers jointly consider acquisition and retention (Jain and Singh 2002), and most of them treat acquisition and retention probabilities as independent (e.g., Gupta, Lehmann, and Stuart 2004; Blattberg and Deighton 1996). This assumption may not accurately reflect the behavior of customers, thereby adversely affecting the firm's forecasts and marketing decisions.

In this research, we develop a joint timing model to explore the acquisition and retention of service by prospective customers of a contractual service provider. Specifically, we consider three aspects of customer behavior in the acquisition and retention of a service. First, we allow for duration dependence in both processes. That is, the likelihood that a prospect acquires a particular service may change as he goes even longer without that service. Similarly, subsequent to service acquisition, the likelihood of discarding the service may also depend on how long the customer has subscribed to that service. Next, we account for customer heterogeneity, as households have different propensities for acquiring and discarding a particular service. Lastly, we incorporate the notion of correlated processes - that is, the correlation that may exist between the acquisition and retention durations, and the parameters that govern them.

While customer heterogeneity and duration dependence have received much attention in extant timing models (e.g., Morrison and Schmittlein 1980), limited research has focused on their role in the acquisition and retention processes. To illustrate the importance of jointly 
considering acquisition and retention, consider two prospects, $\mathrm{A}$ and $\mathrm{B}$, for a contractual service provider, depicted in Figure 1.

\section{[Insert Figure 1]}

The times that elapsed between the beginning of acquisition efforts for prospects A and B and when they acquired service are denoted $t_{A}$ and $t_{B}$, respectively, and the durations for which they maintained service are given by $d_{A}$ and $d_{B}$, respectively. Based on these observations, should the firm pursue those prospects who have gone without the service for longer periods of time? If there were no relationship between the time until acquisition and length of service retention, the company would expect both prospects to retain service for the same length of time. However, it may be the case (as depicted in Figure 1) that prospects like A, who are slow to acquire service, maintain it for a longer period of time (e.g., "cautious but loyal"), whereas prospects who acquire service quickly (like prospect B) also discard it quickly (e.g., "hit and run").

We can broadly classify potential relationships between the acquisition and retention processes into three types. First, a negative relationship may exist between time until acquisition and retention duration. As such, customers who were slow to acquire a service would not be expected to retain service as long as a customer who acquired it earlier. If true, the firm may want to devote resources toward acquiring "younger" prospects rather than "older" prospects based on their expected tenure. On the other hand, in the case of a positive relationship (as reflected in Figure 1), customers who acquired a particular service later will have a longer expected tenure. In this scenario, "older" prospects may be tempting targets. Even though they have not yet acquired service, if they do, they will be expected to retain service longer, and therefore may generate greater long-term revenue, than early acquirers. Finally, there may be no relationship between acquisition and retention duration, as is commonly assumed. As such, the 
time at which prospective customers acquire a service is uninformative of their tenure and targeting plays no significant role. Providing a modeling framework within which to understand the relationship that exists between the time until acquisition and length of service retention is one of the objectives of this research.

In exploring the relationship between acquisition of service and duration of retention, we consider two possible sources of correlation that can exist. First, we allow for correlation in the latent adoption and retention propensities of a household by employing latent classes, where each latent class may have different acquisition and retention behaviors (e.g., Kamakura and Russell 1989). Latent classes alone, however, will only account for correlation in the acquisition and retention propensities across customers. That is, given a particular household's class membership, the two processes are assumed to be independent. However, the time of acquisition and retention durations may also be linked within each latent class. To model this "double correlation" structure, we make use of the Sarmanov family of multivariate distributions (Kotz, Balakrishnan and Johnson 2000; Lee 1996) in conjunction with the latent class framework.

Previous marketing applications of the Sarmanov family of distributions have focused on noncontractual settings (Danaher 2006; Danaher and Hardie 2005; Park and Fader 2004) in contrast with the contractual nature of the present setting. Furthermore, none of these previous studies, accounted for time-varying covariates, such as promotional activity. In addition to applying the Sarmanov family of distributions to contractual data, our work provides a methodological contribution by demonstrating a general approach for incorporating time-varying covariates into a bivariate Sarmanov distribution.

In considering the time until acquisition and subsequent duration of service, the need to account for censoring is highlighted. Many prospective customers may not acquire a particular 
service during the observation period, yet might acquire the service after the observation period ends (right censoring of the acquisition process). Of those customers who do acquire service, some customers may retain it through the end of the observation period but drop service at a later point in time (right censoring of the retention process). As a result, standard bivariate analyses, such as computing the correlation between observed acquisition and retention times, may require the discarding of a significant portion of the observed data (i.e., those prospects who do not acquire service, as well as those who acquire service but maintain it through the observation period). Our joint stochastic model provides a natural way to account for censored observations via the inclusion of survival functions for both processes in the likelihood.

The remainder of this paper proceeds as follows. In Section II, we review existing literature related to the acquisition and retention of services. Section III discusses the data employed in our empirical analysis. In Section IV, we present the development of the proposed joint timing model. The empirical analysis is detailed in Section V, including a discussion of a series of models that are estimated. The results of our analysis are presented in Section VI. Managerial implications and directions for future research are discussed in Section VII.

\section{Previous Studies on Acquisition and Retention}

Despite the importance of considering both acquisition and retention as part of a comprehensive customer valuation model, relatively few studies have taken both of them into account. Those that do deal with both processes often assume complete independence across them. For instance, Gupta, Lehmann, and Stuart (2004) propose a rich framework for determining the value of an entire customer base, but they assume that the acquisition and retention processes are independent. Blattberg and Deighton (1996) discuss the need for firms to balance their marketing expenditures between acquiring new customers and retaining existing 
customers. They propose a framework to achieve an appropriate balance across acquisition spending and retention spending, but they do not consider any links that may exist between the two processes. Berger and Nasr-Bechwati (2001) apply a similar approach to the problem of resource allocation, but like Blattberg and Deighton (1996), they do not shed light on the underlying relationship in acquisition and retention that may exist at the household level.

Perhaps the first paper to consider separate models for each process is Hansotia and Wang (1997), who discuss the importance of acquiring those customers who will be most profitable on the basis of their lifetime value. Their treatment of lifetime value, however, considers the binary acquisition process (yes/no) as independent of the retention process, which is modeled using a right-censored Tobit model.

The most significant contribution in this area is that of Thomas (2001), who proposes a methodology for linking the customer acquisition and retention processes that is closest in spirit to our model, incorporating heterogeneity and correlation between the acquisition and retention processes, yet is still substantially different from our approach. Thomas employs a Tobit model with selection to jointly model the acquisition and retention of an optional membership available to individuals who already belong to an organization. This model is tantamount to employing a binary (yes/no) probit model for the customer's decision to acquire membership and a Tobit model with right-censoring to model the time for which he retains it, where the errors for the probit and Tobit models are correlated. Latent classes are employed to incorporate heterogeneity into the acquisition and retention processes.

Reinartz, Thomas, and Kumar (2005) extend Thomas' (2001) earlier model to simultaneously model acquisition, retention, and customer profitability. Actions taken by the firm and customer, as well as customer characteristics, are assumed to affect the acquisition 
process, the retention process, and customer profitability. The three outcome variables (acquisition, duration, and profitability) are assumed to have a correlated error structure. The authors explore how the level of investment and resource allocation between acquisition and retention can differentially affect customer acquisition, retention, and profitability. As does Berger and Nasr-Bechwati (2001), this research emphasizes the need to jointly model acquisition and retention for marketing decisions.

While Thomas (2001) and Reinartz, Thomas, and Kumar (2005) account for the link between acquisition and retention (through correlated errors), there are several major limitations to the generalizability of their approach. First, acquisition is considered as a binary variable. Consequently, only the decision to acquire service (yes/no) is modeled, rather than the time at which acquisition occurred. As such, these models assume that retention duration and profitability do not depend on the time of acquisition. There are, however, several situations, including service acquisition (which is the context of our empirical application), in which the time that has elapsed since a household came under observation and when it actually acquired the particular service may be known. For example, households may come under the observation of firms via rented mailing lists (e.g., Bitran and Mondschein 1996) or through opt-in activities (e.g., Milne and Rohm 2000). The length of this acquisition period may convey information about subsequent activity, such as retention (in a contractual setting) or purchasing (in a transactional setting), which may offer insights about the value of prospective customers.

These models also do not facilitate the incorporation of duration dependence or timevarying covariates into the acquisition/retention process. Prospective customers may become more or less likely to acquire (or discard) service over time. In addition, the company may use promotional activity to entice customers to acquire a particular service, the effect of which may 
accumulate over time. This will also affect customers' likelihood of retaining service while the promotion is active. In this research, we use the proportional hazards framework (which easily accommodates both duration dependence and time-varying covariates) to construct the marginal acquisition and retention processes and then allow for correlation between the processes.

In summary, the current research builds upon previous literature for the purpose of developing a joint timing model of service acquisition and duration of service (retention). As previous research has demonstrated the importance of considering both acquisition and retention simultaneously, we build a household-level model that allows us to explore the possible underlying relationship between these two processes. In addition, unlike previous research, our methodology allows for us to incorporate duration dependence and time-varying covariates into the model, as well as to examine the "double correlation" that may exist between acquisition and retention processes at the household level. Understanding this link will allow firms to determine if prospective customers are worth the resources needed to convert them to active customers.

\section{Data}

Monthly subscription data were provided by a major provider of telecommunications services. From the subscription records, we constructed a new data set by sampling half of the customers (who began service with the firm between February 2002 and September 2002) for calibration and used the remaining half for a holdout analysis. These customers were observed from the time at which they started service through May 2004. To demonstrate the proposed methodology, we focus on those customers who did not initially subscribe to HBO service, but came under observation as an $\mathrm{HBO}$ prospect by subscribing to other services (e.g., high-speed Internet or digital cable). There were 6211 households in the calibration sample and 6236 households in our holdout sample. 
Time until acquisition was computed by counting the number of months that elapsed between the household's first month of (non-HBO) service with the provider and when the customer, if ever, added HBO. Note that time until acquisition may be right-censored, as some households will not acquire the service during the observation period. In a similar vein, the duration for which a customer retains HBO service was computed by counting the number of months for which a customer keeps service following its acquisition. Retention times may also be right-censored. ${ }^{1}$

In the calibration sample, $84.1 \%$ of prospects did not acquire service. In Table 1 , we provide an overview of the acquisition and retention behavior by $15.9 \%$ of the prospects in the calibration sample who did acquire service.

\section{[Insert Table 1]}

There are three particular features of the data that are worth noting. First, the majority of households do not acquire service during the observation period. As Thomas (2001) discusses, analyses that ignore censored data will therefore yield misleading results. Second, of those households that do acquire HBO service, most acquire service early on. Taken together, these observations seem to indicate that negative duration dependence is present in the acquisition process. Lastly, the retention behavior appears to vary between those prospects who acquired service early (before 12 months) and those who acquired later (after 12 months). Of those prospects who acquired service early, the majority (81\%) discard service within 12 months of acquisition. In contrast, only $51 \%$ of later acquirers discard service during their first 12 months, indicating a possible positive relationship between time of acquisition and duration of retention. But, is this difference attributable to difference across households or correlation between the two

\footnotetext{
${ }^{1}$ There are a limited number of households who discard and re-subscribe to service. In our model, we restrict attention to the initial acquisition and retention spells.
} 
processes? We now turn to the development of the proposed joint timing model, which will allow us to address this important question.

\section{Model Development}

In this section, we build a continuous-time joint model for the acquisition and retention of a service that incorporates duration dependence, unobserved heterogeneity, and correlation in the two processes, and demonstrate how it can be adapted to discrete-time (e.g., monthly) data.

IV.1 A Household-Level Bivariate Model of Acquisition and Retention Behavior

Our model considers the acquisition and retention of a particular service for prospective customers who have come under observation. We begin by assuming that households acquire service according to a Weibull distribution with parameters $\lambda_{s}$ and $\alpha_{s}$, where the probability of acquiring service at time $t_{h}$, conditional on belonging to latent segment $s$, is given by:

$$
f\left(t_{h} \mid s, \lambda_{s}, \alpha_{s}\right)=S_{A}\left(t_{h}-1 \mid s, \lambda_{s}, \alpha_{s}\right)-S_{A}\left(t_{h} \mid s, \lambda_{s}, \alpha_{s}\right) \text { for } t_{h}=1,2, \ldots
$$

where $S_{A}(x)$ is given by the survival function of a Weibull distribution:

$$
S_{A}\left(t_{h} \mid s, \lambda_{s}, \alpha_{s}\right)=e^{-\lambda_{s}\left(t_{h}\right)^{\alpha_{s}}}
$$

and $\alpha_{s}$ and $\lambda_{s}$ are parameters specific to latent segment $s$. If the household acquires service during the observation period, $t_{h}$ is the length of time that has elapsed between when household $h$ came under observation and when household $h$ acquired service, and the acquisition censoring variable $c_{h}^{A}=0$. If the household does not acquire service during the observation period, $T_{h}$, let $t_{h}=T_{h}$ and let $c_{h}^{A}=1$.

Once households acquire service, they discard service according to a different Weibull distribution, governed by parameters $\theta_{s}$ and $\gamma_{s}$. The service provider indicated that a three-month introductory promotion (occurring during the month of acquisition and the following two months) was standard. To incorporate the time-varying promotional activity (or covariates, in 
general) into the retention process, we employ a proportional hazard regression in addition to the Weibull baseline hazard function. The hazard function and survival probability, respectively, are given by

$$
h\left(t \mid \theta_{s}, \gamma_{s}, \beta_{s}, \mathbf{X}(t)\right)=\gamma_{s} \theta_{s} t^{\gamma_{s}-1} e^{\beta_{s} \mathbf{x}(t)}
$$

and

$$
S_{R}\left(t \mid \theta_{s}, \gamma_{s}, \beta_{s}, \mathbf{X}(t)\right)=e^{-\sum_{v=1}^{t}\left(\int_{v-1}^{v} h\left(u \mid \theta_{s}, \gamma_{s}, \beta_{s}, \mathbf{X}(t)\right) d u\right)}=e^{-\theta_{s} \sum_{v=1}^{t}\left(\left(v^{\gamma_{s}}-(v-1)^{\gamma_{s}}\right) e^{\beta_{s} \mathbf{X}(t)}\right)}
$$

where $\beta_{s}$ is the impact of the promotional activity, denoted $\mathbf{X}(t)$. The duration of service, $d_{h}$, is therefore distributed as:

(5) $g\left(d_{h} \mid s, \theta_{s}, \gamma_{s}, \beta_{s}, \mathbf{X}(t)\right)=S_{R}\left(d_{h}-1 \mid s, \theta_{s}, \gamma_{s}, \beta_{s}, \mathbf{X}(t)\right)-S_{R}\left(d_{h} \mid s, \theta_{s}, \gamma_{s}, \beta_{s}, \mathbf{X}(t)\right)$

The time at which a household discontinues service is given by $t_{h}+d_{h}$. If the household retains service throughout the observation period, then let $d_{h}=T_{h}-t_{h}$ and the retention censoring variable $c_{h}^{R}=1$; otherwise, let $c_{h}^{R}=0$.

While the service provider offered the same introductory promotion, regardless of time, the same approach could be used to model the effect of time-varying promotional activity on the acquisition process, if any such measures were available. This would be the case if the promotion was not always available, or different types of promotions were available at different times. Within our modeling framework, we could then decompose the effect of promotional activity into two parts: the impact of promotions prior to acquisition (e.g., "How much more likely are prospects to acquire service under the promotion?") and subsequent to service acquisition (e.g., "How much less likely are customers to drop service during the promotion?”).

The Weibull distribution is chosen to model both the acquisition and retention processes because of its flexibility. The hazard rate for the Weibull distribution, which can be loosely 
interpreted as the probability of an event occurring at time $t$ conditional on it not having occurred until time $t$, can be increasing, decreasing, or constant over time. The Weibull distribution thus allows for positive or negative duration dependence, and nests the exponential distribution, which has a constant hazard. This flexibility is desirable in both the acquisition and retention processes. It also yields a closed form hazard function, facilitating the incorporation of timevarying covariates into the modeling framework, making it preferable in duration analysis (Morrison and Schmittlein 1980).

First, consider the acquisition process. The likelihood that a household acquires service may vary as the duration for which the household has not had service increases. If there is positive duration dependence in the acquisition process $\left(\alpha_{s}>1\right)$, households in segment $s$ will be more likely to acquire service the longer that they have gone without it. Conversely, if there is negative duration dependence in the acquisition process $\left(\alpha_{s}<1\right)$, households in segment $s$ will be less likely to acquire service as time passes. When $\alpha_{s}=1$, there is no duration dependence in the acquisition process and it nests the memoryless exponential distribution. The retention process, with Weibull shape parameter $\gamma_{s}$, can be interpreted similarly.

IV.2 Incorporating "Double Correlation" in Acquisition and Retention

The model presented in equations (1)-(5) outlines a flexible bivariate timing model that allows for time-varying covariates. However, it treats time until acquisition and retention duration as independent processes (conditional on membership in latent segment $s$ ). To model the possible correlation between the observed acquisition and retention processes, we employ the Sarmanov family of bivariate distributions (e.g., Lee 1996; Park and Fader 2004). Let the function $f(x, y)$ be defined as:

$$
f(x, y)=f_{x}(x) \times f_{y}(y) \times\left\{1+\omega \phi_{x}(x) \phi_{y}(y)\right\}
$$


where $f_{x}(x)$ and $f_{y}(y)$ are univariate probability density functions, and $\phi_{x}(x)$ and $\phi_{y}(y)$ are bounded mixing functions such that $\int_{-\infty}^{\infty} f_{z}(z) \phi_{z}(z) d z=0$ for $z=x, y$. In order for $f(x, y)$ to be a bivariate density function, $\phi_{x}(x), \phi_{y}(y)$, and $\omega$ must satisfy the condition $1+\omega \phi_{x}(x) \phi_{y}(y) \geq 0$ for all values of $x$ and $y$. As such, $\omega$ can be interpreted as the unnormalized correlation between $f_{x}(x)$ and $f_{y}(y)$.

While there are other families of bivariate distributions generated from the combination of two univariate distributions (e.g., Farlie 1960; Johnson and Kotz 1975, 1977), one of the benefits that the Sarmanov family of distributions provides is that the marginal distributions are guaranteed to take on the desired univariate densities. This is the only class of multivariate distributions that offers this property, which greatly aids in the closed-form computation of censored events. Consider a customer who does not acquire service during the observation period. This customer may acquire service after the observation period and then proceed to retain service according to the retention process. To calculate the probability that the customer acquires service after $T_{h}$, we must integrate over all possible unobserved retention durations. By using a Sarmanov bivariate distribution, this marginal likelihood will be equal to the likelihood computed from the univariate acquisition distribution, which is directly available.

Lee (1996) demonstrates how to find the mixture functions for different distributions $f_{x}(x)$ and $f_{y}(y)$. Lee shows that the mixing distribution for any univariate density function $f(x)$ is given by:

$$
\phi(x)=f(x)-\int_{-\infty}^{\infty} f^{2}(t) d t
$$

Replacing the integral in equation (7) with a summation provides the general form of the mixing function for a discrete distribution $f(x)$. Then, replacing $f(x)$ in equation (7) with the probability 
mass functions given in equations (1) and (5) yields the mixing functions for the acquisition and retention processes, denoted $\phi_{A}(x)$ and $\phi_{R}(y)$, respectively:

$$
\begin{gathered}
\phi_{A}\left(x \mid \lambda_{s}, \alpha_{s}\right)=S_{A}\left(x-1 \mid \lambda_{s}, \alpha_{s}\right)-S_{A}\left(x \mid \lambda_{s}, \alpha_{s}\right) \\
+2 \sum_{i=1}^{\infty}\left[S_{A}\left(i \mid \lambda_{s}, \alpha_{s}\right)\left(S_{A}\left(i-1 \mid \lambda_{s}, \alpha_{s}\right)-S_{A}\left(i \mid \lambda_{s}, \alpha_{s}\right)\right)\right]-1
\end{gathered}
$$

and

$$
\begin{gathered}
\phi_{R}\left(y \mid \theta_{s}, \gamma_{s}, \beta_{s}, \mathbf{X}(t)\right)=S_{R}\left(y-1 \mid \theta_{s}, \gamma_{s}, \beta_{s}, \mathbf{X}(t)\right)-S_{R}\left(y \mid \theta_{s}, \gamma_{s}, \beta_{s}, \mathbf{X}(t)\right) \\
+2 \sum_{i=1}^{\infty}\left[S_{R}\left(i \mid \theta_{s}, \gamma_{s}, \beta_{s}, \mathbf{X}(t)\right)\left(S_{R}\left(i-1 \mid \theta_{s}, \gamma_{s}, \beta_{s}, \mathbf{X}(t)\right)-S_{R}\left(i \mid \theta_{s}, \gamma_{s}, \beta_{s}, \mathbf{X}(t)\right)\right)\right]-1
\end{gathered}
$$

The joint distribution of acquisition time and duration of service, conditional on the class $s$ to which the household belongs, is then given by:

$$
\begin{aligned}
j\left(t_{h}, d_{h} \mid s, \lambda_{s}, \alpha_{s}, \theta_{s}, \gamma_{s}, \beta_{s}, \mathbf{X}(t)\right)=f\left(t_{h} \mid s, \lambda_{s}, \alpha_{s}\right) g\left(d_{h} \mid s, \theta_{s}, \gamma_{s}, \beta_{s}, \mathbf{X}(t)\right) \\
\times \\
\times\left\{1+\omega_{s} \phi_{A}\left(t_{h} \mid s, \lambda_{s}, \alpha_{s}\right) \phi_{R}\left(d_{h} \mid s, \theta_{s}, \gamma_{s}, \beta_{s}, \mathbf{X}(t)\right)\right\}
\end{aligned}
$$

The household-level joint timing model given in equation (10) relaxes the assumption of independence between the two processes and allows for correlation in acquisition and retention. This, however, may not be the only type of relationship that exists between the two processes. In addition to a correlation in the observed durations (as detailed in equations (6)-(10)), there may also be correlation in the latent acquisition and retention propensities. Park and Fader (2004) refer to the correlation in the underlying parameters as linked propensities.

To account for linked propensities, we employ a latent class model with $s$ unobserved classes (e.g., Kamakura and Russell 1989; Thomas 2001). In addition to modeling the unobserved heterogeneity in household's propensities to acquire and discard service, as the parameters governing both the acquisition and retention processes are segment-specific, the latent classes also allow us to capture the linked propensities. For example, one latent class may be characterized by high acquisition likelihood and high retention likelihood, whereas another 
may be marked by high acquisition likelihood and low retention likelihood. The latent classes may also differ with regards to the direction in (and speed with) which these likelihoods change over time (duration dependence), the correlation between acquisition and retention processes (governed by $\left.\omega_{\mathrm{s}}\right)$, and the sensitivity to the promotional activity $\left(\beta_{\mathrm{s}}\right)$.

\section{IV.3 Accounting for Censored Observations}

Due to the potential right censoring of acquisition and retention times, we can categorize customers into three groups based on whether or not they acquire service and, if they acquire it, whether or not they discard service during the observation period. The first group is comprised of those customers who do not acquire the particular service during their observation period $\left(c_{h}^{A}=1\right)$. The time until acquisition for these customers therefore lies in the interval $t_{h} \in\left(T_{h}, \infty\right)$.

If household $h$ acquires service after $T_{h}$, it may retain service for any length of time $d_{h}$.

Therefore, the probability that household $h$ does not acquire service by time $T_{h}$, conditional on his segment membership, using the discrete-time bivariate Sarmanov Weibull distribution, is given by:

$$
\begin{gathered}
p_{1 s}\left(t_{h}, d_{h} \mid s, \lambda_{s}, \alpha_{s}, \theta_{s}, \gamma_{s}, \beta_{s}, \mathbf{X}(t)\right) \\
=\sum_{x=T_{h}+1}^{\infty} \sum_{y=1}^{\infty} j\left(x, y \mid s, \lambda_{s}, \alpha_{s}, \theta_{s}, \gamma_{s}, \beta_{s}, \mathbf{X}(t)\right) \\
=S_{A}\left(T_{h} \mid s, \lambda_{s}, \alpha_{s}\right)
\end{gathered}
$$

which follows from the property of the Sarmanov mixing function that $\int_{-\infty}^{\infty} f(z) \phi(z) d z=0$. As expected, the probability that a customer does not acquire service during the observation period is given by the survival probability of the univariate acquisition distribution.

The next group consists of those households that acquire service within and maintain it through the observation period $\left(c_{h}^{A}=0, c_{h}^{R}=1\right)$. The probability that household $h$ starts service at time $t_{h}$ and maintains it through the observation period is given by: 


$$
\begin{aligned}
& p_{2 s}\left(t_{h}, d_{h} \mid s, \lambda_{s}, \alpha_{s}, \theta_{s}, \gamma_{s}, \beta_{s}, \mathbf{X}(t)\right) \\
= & \sum_{y=d_{h}+1}^{\infty} j\left(t_{h}, y \mid s, \lambda_{s}, \alpha_{s}, \theta_{s}, \gamma_{s}, \beta_{s}, \mathbf{X}(t)\right)
\end{aligned}
$$

Lastly, there are households who acquire the service and subsequently discard it during the observation period $\left(c_{h}^{A}=0, c_{h}^{R}=0\right)$. In this case, neither the acquisition nor the retention process is right censored. Thus, the probability that household $h$ starts service at $t_{h}$ and maintains service for a duration of $d_{h}$ is:

$$
\begin{aligned}
& p_{3 s}\left(t_{h}, d_{h} \mid s, \lambda_{s}, \alpha_{s}, \theta_{s}, \gamma_{s}, \beta_{s}, \mathbf{X}(t)\right) \\
& =j\left(t_{h}, d_{h} \mid s, \lambda_{s}, \alpha_{s}, \theta_{s}, \gamma_{s}, \beta_{s}, \mathbf{X}(t)\right)
\end{aligned}
$$

The household-level likelihood, conditional on the latent segment to which the household belongs, can then be written as:

$$
L^{*}\left(\lambda_{s}, \alpha_{s}, \theta_{s}, \gamma_{s}, \beta_{s} \mid s, t_{h}, d_{h}, c_{h}^{A}, c_{h}^{R}, T_{h}, \mathbf{X}(t)\right)=\left\{\begin{array}{c}
p_{1 s}\left(t_{h}, d_{h}\right), \text { if } c_{h}^{A}=1 \\
p_{2 s}\left(t_{h}, d_{h}\right), \text { if } c_{h}^{A}=0 \text { and } c_{h}^{R}=1 \\
p_{3 s}\left(t_{h}, d_{h}\right), \text { if } c_{h}^{A}=0 \text { and } c_{h}^{R}=0
\end{array}\right.
$$

The unconditional likelihood is given by:

(15) $L\left(\boldsymbol{\lambda}, \boldsymbol{\alpha}, \boldsymbol{\theta}, \boldsymbol{\gamma}, \boldsymbol{\beta} \mid t_{h}, d_{h}, c_{h}^{A}, c_{h}^{R}, T_{h}, \mathbf{X}(t)\right)=\sum_{s=1}^{S} q_{s} L^{*}\left(\lambda_{s}, \alpha_{s}, \theta_{s}, \gamma_{s}, \beta_{s} \mid s, t_{h}, d_{h}, c_{h}^{A}, c_{h}^{R}, T_{h}, \mathbf{X}(t)\right)$

where $\boldsymbol{\lambda}, \boldsymbol{\alpha}, \boldsymbol{\theta}, \boldsymbol{\gamma}$, and $\boldsymbol{\beta}$ denote the vectors of segment-specific parameters and $q_{s}$ denotes the probability that a household is in segment $s$, such that $\sum_{s=1}^{S} q_{s}=1$. Letting $Y_{h}$ denote the observed data vector $\left\{t_{h}^{A}, t_{h}^{R}, c_{h}^{A}, c_{h}^{R}, T_{h}\right\}$, the overall log-likelihood is:

$$
L L\left(\boldsymbol{\lambda}, \boldsymbol{\alpha}, \boldsymbol{\theta}, \boldsymbol{\gamma}, \boldsymbol{\beta} \mid Y_{1}, \ldots, Y_{H}\right)=\sum_{h \in H} \log \left(L\left(\boldsymbol{\lambda}, \boldsymbol{\alpha}, \boldsymbol{\theta}, \boldsymbol{\gamma}, \boldsymbol{\beta} \mid Y_{h}\right)\right)
$$

IV.4 An Illustration of Correlated Processes 
As many readers may be unfamiliar with the Sarmanov family of distributions, we demonstrate the impact of correlated processes by computing the expected retention durations (Fader and Hardie 2006) conditional on the time of acquisition for different values of $\omega$ using the following parameters without loss of generality: $\lambda=.5, \alpha=1, \theta=.5, \gamma=1$, and $\beta=-1{ }^{2}$ The expected tenure, which is a function of a customer's time of acquisition $t_{h}$, is given by:

$$
E T\left(t_{h}\right)=\sum_{d=0}^{\infty} \frac{p_{2 s}\left(t_{h}, d\right)}{f\left(t_{h}\right)}
$$

where the numerator has the same form as $p_{2 s}\left(t_{h}, d\right)$ from equation (12) and the denominator is the marginal probability of acquiring service at time $t_{h}$. In Figure 2, we show the expected tenure of service for subscribers who acquire service in months $1,2,3$, and 4 for the full range of values that $\omega$ can assume.

\section{[Insert Figure 2]}

Under these parameters, a customer beginning service after one month may be expected to retain service up to $33.3 \%$ longer than a customer beginning after four months (at the minimum value of $\omega$ ) or have an expected tenure $34.4 \%$ shorter than that of a customer beginning after four months (at the maximum value of $\omega$ ). Note that the direction of the relationship changes as $\omega$ changes sign. At $\omega=0$, the expected tenure of service is independent of acquisition time. Based on the strength and direction of the relationship between acquisition and retention, customers who acquire service at different times may have different expected tenures and therefore may be of differential value to the service provider.

Also, note that the difference in expected tenure is proportionately greater between households who acquire service in month one and two in comparison to the difference in

\footnotetext{
${ }^{2}$ For these particular parameters, the range of possible correlations is from -0.14 to 0.22 . The narrow range of correlations, however, is deceptive; as can be seen in Figure 2, the correlation exhibits a noticeable effect on the expected retention behavior based on time of acquisition, even over this small range.
} 
expected tenure between those who acquire in month three and four. This pattern continues to hold for all observed acquisition times. Thus, the impact of the correlated processes appears to plateau as the acquisition time increases.

Depending on the nature of the relationship, as reflected by the value of $\omega$, and the type of duration dependence in both the acquisition and retention processes, several behavioral patterns may emerge. For example, a "worst case" scenario would include a negative correlation between acquisition and retention, negative duration dependence in the acquisition process, and positive duration dependence in the retention process. Not only are prospects less likely to acquire the service over time, but, once they acquire it, they are increasingly likely to discard it. Compounding this situation is the negative correlation, which implies that prospects who acquire the service later will not keep the service as long. Here, firms must recognize that it may not be worthwhile to devote resources to prospects who have not acquired the service after some time, if this were to hold.

Prospects who have foregone the service for a long period can, however, be valuable under different conditions. Positive duration dependence in the acquisition process and negative duration dependence in the retention process would yield a scenario in which prospects are increasingly likely to acquire service and decreasingly likely to discard it after acquisition. Add to that hypothetical positive correlation between the acquisition and retention times, and later acquirers will be expected to maintain service for a longer duration than early acquirers. As such, it may be worthwhile to spend a little more to induce acquisition based on how long they will keep service.

\section{Empirical Analysis}


The full model presented in equations (1) - (16) was fit using maximum likelihood estimation in MATLAB. We estimate models with different numbers of latent classes to assess how many classes are needed, based on BIC. In addition, we estimate a series of models in which we assume that $\omega=0$. In these models, we allow for correlation in the parameters governing the acquisition and retention processes across latent classes, but assume that the processes are conditionally independent. To assess the performance of the model on the holdout sample, we examine tracking plots of both marginal processes, acquisition and retention. In addition, we compute the overall mean absolute error (MAE). The MAE was computed by taking the absolute difference in the observed and expected number of prospects with acquisition and retention times $(t, d)$, and then average across all possible pairs of $(t, d)$. This includes both households who did and did not acquire service during the observation period.

\section{Model Results}

\section{VI.1 Empirical Results}

The in-sample BIC and out-of-sample overall MAE are presented in Table 2.

\section{[Insert Table 2]}

First, let us focus on the models that involve a single class; that is, all customers are assumed to be homogenous. On the basis of both BIC (15512 for model 1 vs. 15516 for model 2) and a likelihood ratio test (model $1 \mathrm{LL}=-7736$ vs. model $2 \mathrm{LL}=-7730, \mathrm{p}<.01$ ), we see that the model that allows for correlation between the acquisition and retention processes performs better than the model that assumes the two processes are independent (both in- and out-of-sample). The model parameters are presented in Table 3 . In addition to the parameter estimates, to indicate the strength of the correlation between processes, we compute the percentage of the maximum possible correlation in the direction that $\hat{\omega}$ reflects (if $\hat{\omega}>0$, this is the percentage of the 
maximum possible positive correlation, whereas this is the percentage of the maximum (in absolute terms) possible negative correlation for $\hat{\omega}<0$ ).

\section{[Insert Table 3]}

Note that in both models, there is negative duration dependence in both the acquisition and retention processes $(\hat{\alpha}<1, \hat{\gamma}<1)$. As expected, during the promotional period, subscribers are less likely to discard service $(\hat{\beta}<0)$. Based on $\hat{\omega}$, there is a strong positive correlation between the time until acquisition and the duration of retention; that is, customers who acquire service later keep the service longer.

Next, we move to the models that incorporate heterogeneity via latent classes. Recall that Table 1 indicated a potentially positive relationship between acquisition time and duration of service retention. In the absence of heterogeneity (models 1 and 2), this relationship is attributed to correlation in the two processes. When we allow for latent classes, a different explanation emerges. Based on BIC, allowing for correlated durations in models with 2 and 3 latent classes (Models 3 and 5, respectively) does not significantly improve the fit of the model over the latent class models that ignore correlated durations (Models 4 and 6, respectively). Thus, the observed relationship between time of acquisition and retention duration is more likely attributable to heterogeneity across individuals, rather than correlated durations.

It is important to understand whether the observed associations (between acquisition and retention) are more attributable to correlated propensities versus correlated outcomes. Park and Fader (2004), though in a different context, found more strength in the latter than in the former. Here, we find the opposite result, demonstrating the value of employing a modeling framework that can distinguish between the two, as the implications that one draws (as seen in Table 2) can be quite different. One possible explanation for this difference is that we examine acquisition 
and retention durations of an individual service from a single provider, while Park and Fader (2004) examined visiting behavior at two (competing) retailers.

On the basis of both BIC and the out-of-sample MAE, the two-class model without correlated processes (model 4) is the "winning" model. The model indicates that there are two latent classes of customers present. In contrast to the models that omitted latent classes, the retention process in both classes is marked by positive duration dependence $(\hat{\gamma}>1)$. That is, once we account for differences across customers who have acquired service, they are more likely to discard service as time passes. In addition, we also find that the latent classes respond differently to the promotional activity. While the coefficients may seem to indicate that the second latent class is more sensitive to the promotional activity ( $\hat{\beta}_{2}=-12.23$ vs. $\left.\hat{\beta}_{1}=-0.68\right)$, it is actually the first class that is more responsive. In the first class of customers, the proportion of subscribers who retain service through the promotional period is estimated to be $29 \%$ greater than the proportion would be if the promotion were not offered; in the second class, it is only $5 \%$ greater. If the provider were able to identify the segment to which the household belong, it may be beneficial to selectively offer the promotion.

We next explore the behavior of the two latent segments in regards to both the acquisition and retention processes. In Figure 3a, we show the expected acquisition process for each latent class; the expected retention process is shown in Figure $3 \mathrm{~b}$.

[Insert Figure 3a and 3b]

As discussed earlier, the behavior of the two classes varies greatly in both the acquisition and retention processes. The first class of prospective customers (indicated by the dashed line) acts quickly in terms of both acquisition and retention behavior. By the end of the observation period, nearly $90 \%$ of prospects in this class are expected to have acquired service. Of those 
prospects who do acquire service, almost all are expected to discard service within 12 months.

The second class of prospects (indicated by the dotted line), on the other hand, is both slow to acquire service (fewer than $10 \%$ of this class is expected to acquire service by the end of the observation period) and slow to discard it. At first glance, it would appear that prospects in this segment are worth attracting based on their retention behavior. In the next section, we demonstrate a method to quantify the value of prospects, taking into account how long it takes for them to acquire service.

As the overall MAE only provides a relative measure of model performance, to demonstrate the accuracy of the proposed model on the acquisition and retention aspects, we present tracking plots of both acquisition and retention in Figure 4. In doing so, we can assess the accuracy of the proposed model on the calibration sample, as well as the holdout sample which consists of a completely different set of households. In Figure 4a, we present the cumulative acquisition in the calibration and holdout samples, as well as the expected cumulative acquisition by our proposed model. Similarly, we present the observed retention behavior in the calibration and holdout samples, along with the expected retention curve, in Figure 4b. In this plot, we also present the results of the Tobit with selection model using two latent classes (Thomas 2001). ${ }^{3}$

[Insert Figure 4a and 4b]

With just two classes, the joint Weibull timing model captures the underlying customer behavior in both the acquisition and retention processes. The model fairly accurately tracks the marginal acquisition and retention processes for the calibration sample. For the holdout sample,

\footnotetext{
${ }^{3}$ Like Thomas (2001), in estimating this model, we only utilize the retention duration of individuals who acquired service by the end of the observation period. We note that the Tobit cannot be directly compared to the proposed model, as it only models the retention aspect, whereas we jointly model both retention and acquisition.
} 
the model underpredicts the cumulative adoption after the first month, but the maximum absolute difference between observed and expected cumulative adoption is less than $2 \%$ (of the sample).

Comparing the model fit of the joint Weibull model for observed retention with a twoclass Tobit with selection model, Figure $4 \mathrm{~b}$ reveals that the joint Weibull model is extremely accurate during both the calibration and holdout periods. While the maximum absolute difference in the proportion of subscribers retaining service, between the observed and expected under the proposed model, is less than $2 \%$ (of subscribers), the Tobit with selection model has a maximum absolute difference of $11 \%$. Not only does the proposed model more accurately estimate retention than the Tobit with selection model, but it also allows us to understand how the retention process (and subsequent measures, such as expected tenure) changes based on the time of acquisition.

\section{VI.2 Computing the Discounted Expected Tenure}

As $\omega=0$ in the chosen model (model 4 ), the expected tenure, conditional on class membership, does not depend on the time of acquisition. For a customer in class 1, using equation (17), the expected tenure is 4.42 months. For a customer in class 2 , the expected tenure is 24.75 months. These estimates, however, are conditional on the customers having acquired service. But, will later subscribers actually be more valuable to the service provider given that it will take longer to acquire them, or should the service provider instead focus on retaining current subscribers?

To address these questions, we compute the discounted expected tenure (DET) of customers given their acquisition activity. Analogous to Fader, Hardie and Lee's (2005) calculation of discounted expected transactions, our measure assumes that a subscriber today is more valuable than a subscriber tomorrow. To estimate the discounted expected tenure, we 
modify equation (17) to incorporate a discount factor and update our belief of a customer's class membership. At time $t=0$, the discounted expected tenure of a customer who acquires service after being under observation for $t_{h}$ months is given by:

$$
\operatorname{DET}\left(t_{h}\right)=\sum_{s=1}^{S} z_{s}\left(t_{h}\right) \sum_{y=0}^{\infty} \frac{S_{R s}\left(y \mid \theta_{s}, \gamma_{s}, \beta_{s}, \mathbf{X}(t)\right)}{(1+d)^{y+t_{h}}}
$$

The innermost summation is the expected tenure from equation (17), with each month of service discounted from time $t=0 .{ }^{4}$ Then, in the outer summation, $z_{s}$ denotes our updated belief of class membership based on the time of acquisition. Using Bayes' rule, the probability of a household being in class $s$, given that they acquired service in month $t_{h}$, is:

$$
z_{s}\left(t_{h}\right)=\frac{q_{s} f_{s}\left(t_{h}\right)}{\sum_{x=1}^{S} q_{x} f_{x}\left(t_{h}\right)}
$$

where $f_{s}\left(t_{h}\right)$ is the class-specific likelihood of acquiring service at time $t_{h}$, and $q_{s}$ is the estimated proportion of households belonging to class $s$.

In Figure 5, we assume a 15\% annual discount rate and show how the discounted expected tenure changes based on a customer's time of acquisition for a five-year time frame.

\section{[Insert Figure 5]}

Figure 5 shows the posterior DET, as well as the DET conditional on class membership and the DET based on the Tobit model. Note that the DET of the Tobit model monotonically decreases the later prospects acquire service. As it does not account for the time of acquisition, it is only affected by the discounting of the future. While latent classes are employed in both approaches, our proposed model is uniquely able to leverage information from the acquisition process to learn about prospects' expected retention behavior.

\footnotetext{
${ }^{4}$ In model 4, the acquisition and retention processes are conditionally independent, allowing us to use $S_{R}$ from equation (4) rather than the quotient $p_{2}\left(t_{h}, d\right) / f\left(t_{h}\right)$ that is used in equation (17). The latter, when $\omega=0$, nests the former.
} 
In contrast, there are two forces acting upon the posterior DET in our proposed model. First, the later a prospect acquires service, the more likely that he belongs to the second segment of customers. Recall that this segment is both slow to acquire service and slow to discard it; so, later acquirers will have a greater expected tenure. The discounted expected tenure, however, will not increase without bound. While the second class of customers has an expected tenure of 24.75 months, the maximum posterior DET is 9.86 months. While the expected tenure of prospects increases the later that they acquire service (as it becomes more likely that they are in the second class), because a subscriber today is more valuable than a subscriber tomorrow, this increase is tempered by discounting the future. Though late-arriving customers may have a longer expected tenure, they will not acquire service for several months (or years), which diminishes the benefit of the positive relationship between acquisition and retention times.

Once these relationships are clarified in this manner, the resulting acquisition and retention curves can be used as inputs into the kinds of resource allocation tasks that have been discussed by authors such as Blattberg and Deighton (1996). Our estimate of discounted expected tenure can also be incorporated into calculations of customer value, as well as lending itself to use in dynamic programming applications. For example, managers can determine, based on prospects' discounted expected tenures and acquisition costs, when they should cease acquisition activities.

\section{Conclusions and Implications}

We have developed a joint model of the time until acquisition and duration for which customers retain service. Our modeling framework incorporates household-level heterogeneity and duration dependence into both the acquisition and retention processes. In the spirit of Park and Fader (2004), we allow for "double correlation," within and across customers for these two 
processes. While other work has examined the relationship between acquisition (as a yes/no decision) and subsequent retention, to the best of our knowledge, our work is among the first to jointly model acquisition and retention spells in a contractual setting.

Testing for the link between acquisition and retention can have important implications for marketing activities such as customer valuation and resource allocation. If a link exists between the acquisition and retention processes, certain customers may be more valuable to the firm than others, as demonstrated by the example presented earlier. Our analysis reveals two classes of customers, one that is marked by high acquisition propensities but low retention propensities, while the other is characterized by low acquisition probabilities and high retention probabilities.

These segments can be likened to Reinartz and Kumar's (2002) notion of "butterflies and barnacles." The butterfly class is fast-moving, both to acquire and discard service, whereas the barnacle class is slow to act in terms of acquiring and discarding service. Though the barnacles are slow to acquire service, based on their discounted expected tenure, they may be more valuable. However, the benefit of an increased discounted expected tenure must be balanced against the costs of acquiring these customers. If pricing information and the cost of acquisition efforts were available, our measure of discounted expected tenure could be incorporated into a break-even analysis to determine if and when the firm should cease acquisition efforts. It may be the case that later subscribers are less valuable because of the costs associated with inducing them to acquire service.

It is important to recognize, though, that the "butterflies and barnacles" analogy does not only reflect the mean tendency of customers to arrive/leave quickly or slowly; it also reflects the critical (but subtle) role that duration dependence plays in both processes. As researchers such as 
Morrison and Schmittlein (1980) have observed, there are distinct roles for heterogeneity versus duration dependence in these timing models, and both should be formally accommodated.

There are certain limitations to our proposed methodology that must be acknowledged. First, our data covers only one service for one company; future research should consider the interplay of competing services and/or firms in the acquisition and retention of service with a particular provider. Popular notions such as "share of wallet" require extensions to the multiservice and multi-firm setting. Future work should also introduce other marketing variables and customer characteristics. For instance, it would be useful to determine if the mode of acquisition has any impact on the acquisition-retention relationship. It might also make sense to investigate whether there is a dynamic aspect to this relationship: does the acquisition-retention association evolve over time as a service (and its customer base) matures? The future pool of customer prospects might have different acquisition-retention tendencies that the current group.

It is too early to speculate about the existence and nature of such dynamic relationships. But we do hope that other researchers will continue down this path in order to better understand the interplay among the underlying processes that new customers follow as they come to a new service provider and subsequently depart. It is not clear what kinds of substantive observations or "empirical generalization" will arise, but it is important for researchers to use the right tools to uncover and characterize them. We hope that our proposed model will be part of the toolkit for many future investigations. 
Table 1. Overview of Acquisition and Retention Timing for Customers Who First Acquired Service from $2 / 03$ through $9 / 03$.

\section{Retention}

\begin{tabular}{|c|c|c|c|c|}
\hline \multirow{4}{*}{ Acquisition } & $\begin{array}{c}\text { Within 12 months } \\
\text { of acquisition }\end{array}$ & $\begin{array}{c}\text { After 12 months } \\
\text { of acquisition }\end{array}$ & $\begin{array}{c}\text { Right- } \\
\text { censored }\end{array}$ \\
\cline { 2 - 5 } & Before Month 12 & $62.3 \%$ & $5.0 \%$ & $9.4 \%$ \\
\cline { 2 - 5 } & After Month 12 & $12.0 \%$ & 0 & $11.3 \%$ \\
\cline { 2 - 5 }
\end{tabular}


Table 2. Model Results

\begin{tabular}{|c|c|c|c|c|c|}
\hline Model & Classes & $\begin{array}{c}\text { Correlated } \\
\text { Processes? }\end{array}$ & LL & BIC & $\begin{array}{c}\text { Overall } \\
\text { MAE }\end{array}$ \\
\hline 1 & \multirow{2}{*}{1} & Yes & -7730 & 15512 & 4.51 \\
\cline { 3 - 6 } & \multirow{2}{*}{2} & No & -7736 & 15516 & 4.49 \\
\hline 3 & Yes & -7666 & 15445 & 4.27 \\
\hline 4 & No & -7669 & 15435 & 4.24 \\
\hline 5 & \multirow{2}{*}{3} & Yes & -7647 & 15468 & 4.30 \\
\cline { 3 - 6 } & & No & -7652 & 15453 & 4.27 \\
\hline 6 & & \multicolumn{3}{|c}{} \\
\hline
\end{tabular}


Table 3. Model Parameters

\begin{tabular}{|c|c|c|c|c|c|c|c|c|c|}
\hline Model & Classes & $\hat{\lambda}$ & $\hat{\alpha}$ & $\hat{\theta}$ & $\hat{\gamma}$ & $\hat{\beta}$ & $\hat{\omega}$ & $\%$ corr. & $\hat{\boldsymbol{q}}$ \\
\hline 1 & \multirow{2}{*}{1} & 0.04 & 0.45 & 0.38 & 0.67 & -1.24 & 154.2 & $44.27 \%$ & -- \\
\hline 2 & & 0.04 & 0.44 & 0.46 & 0.63 & -1.39 & 0 & 0 & -- \\
\hline \multirow[t]{2}{*}{3} & \multirow{4}{*}{2} & Class 1: 0.27 & 0.66 & 0.22 & 1.22 & -0.58 & 22.5 & $26.5 \%$ & 0.12 \\
\hline & & Class 2: 0.01 & 0.52 & 0.01 & 1.77 & -13.05 & -484.1 & $9.9 \%$ & 0.88 \\
\hline \multirow[t]{2}{*}{4} & & Class 1: 0.27 & 0.65 & 0.23 & 1.20 & -0.68 & 0 & 0 & 0.13 \\
\hline & & Class 2: 0.01 & 0.55 & 0.02 & 1.72 & -12.23 & 0 & 0 & 0.87 \\
\hline \multirow[t]{3}{*}{5} & \multirow{6}{*}{3} & Class 1: 0.03 & 0.58 & 0.17 & 0.53 & -0.36 & 3.95 & $1.0 \%$ & 0.16 \\
\hline & & Class 2: 0.26 & 1.22 & 0.17 & 1.52 & -0.52 & 65.9 & $92.8 \%$ & 0.04 \\
\hline & & Class 3: 0.03 & 0.44 & 0.07 & 2.27 & -1.38 & 61.1 & $26.4 \%$ & 0.80 \\
\hline \multirow[t]{3}{*}{6} & & Class 1: 0.12 & 0.73 & 0.01 & 1.20 & -0.26 & 0 & 0 & 0.05 \\
\hline & & Class 2: 0.07 & 1.84 & 0.18 & 0.83 & -0.05 & 0 & 0 & 0.03 \\
\hline & & Class 3: 0.03 & 0.39 & 0.14 & 1.65 & -0.90 & 0 & 0 & 0.92 \\
\hline
\end{tabular}


Figure 1. Depiction of Acquisition-Retention Relationship



Prospect comes under observation 
Figure 2. Impact of Correlated Processes on Expected Tenure

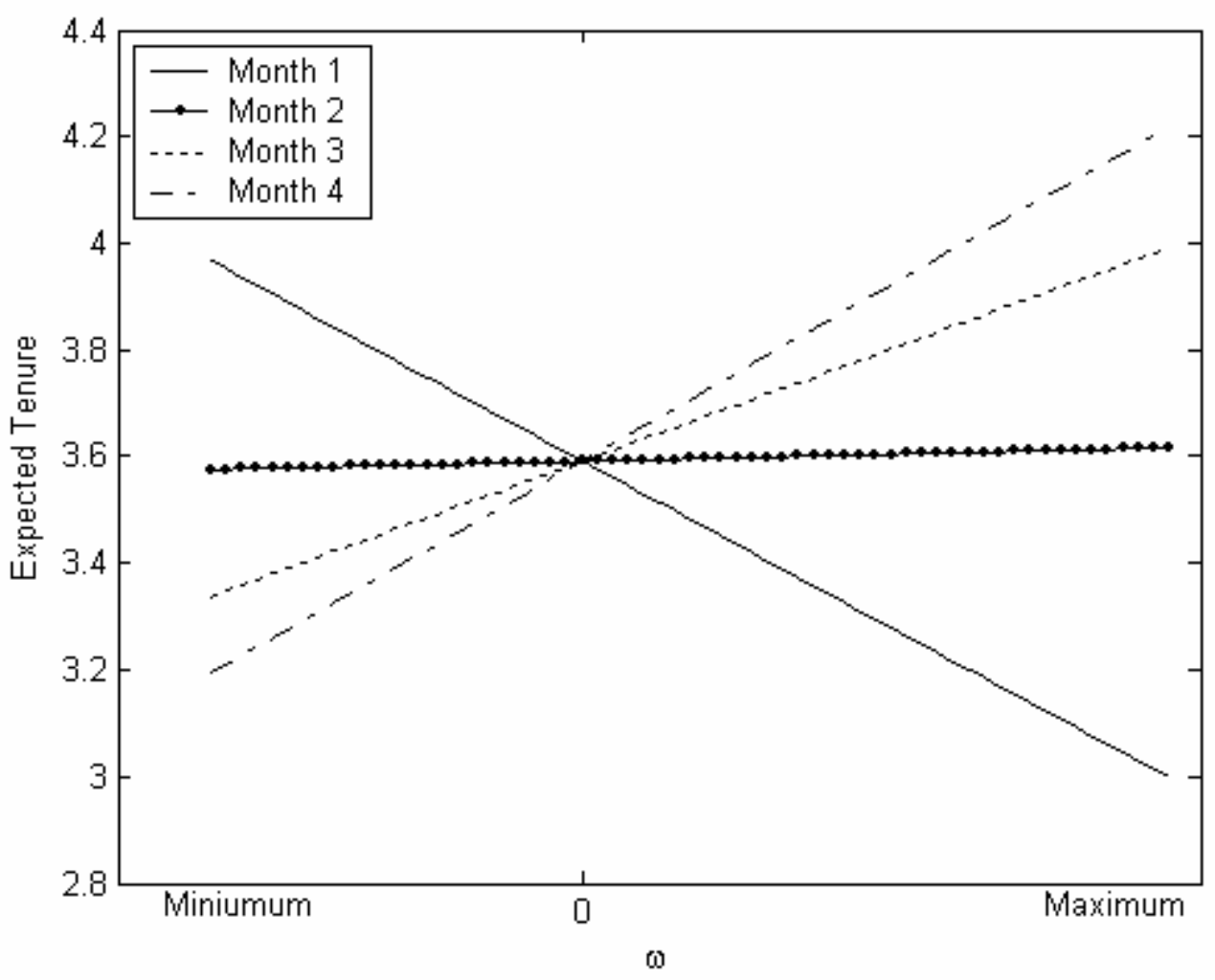


Figure 3a. Cumulative acquisition by latent class

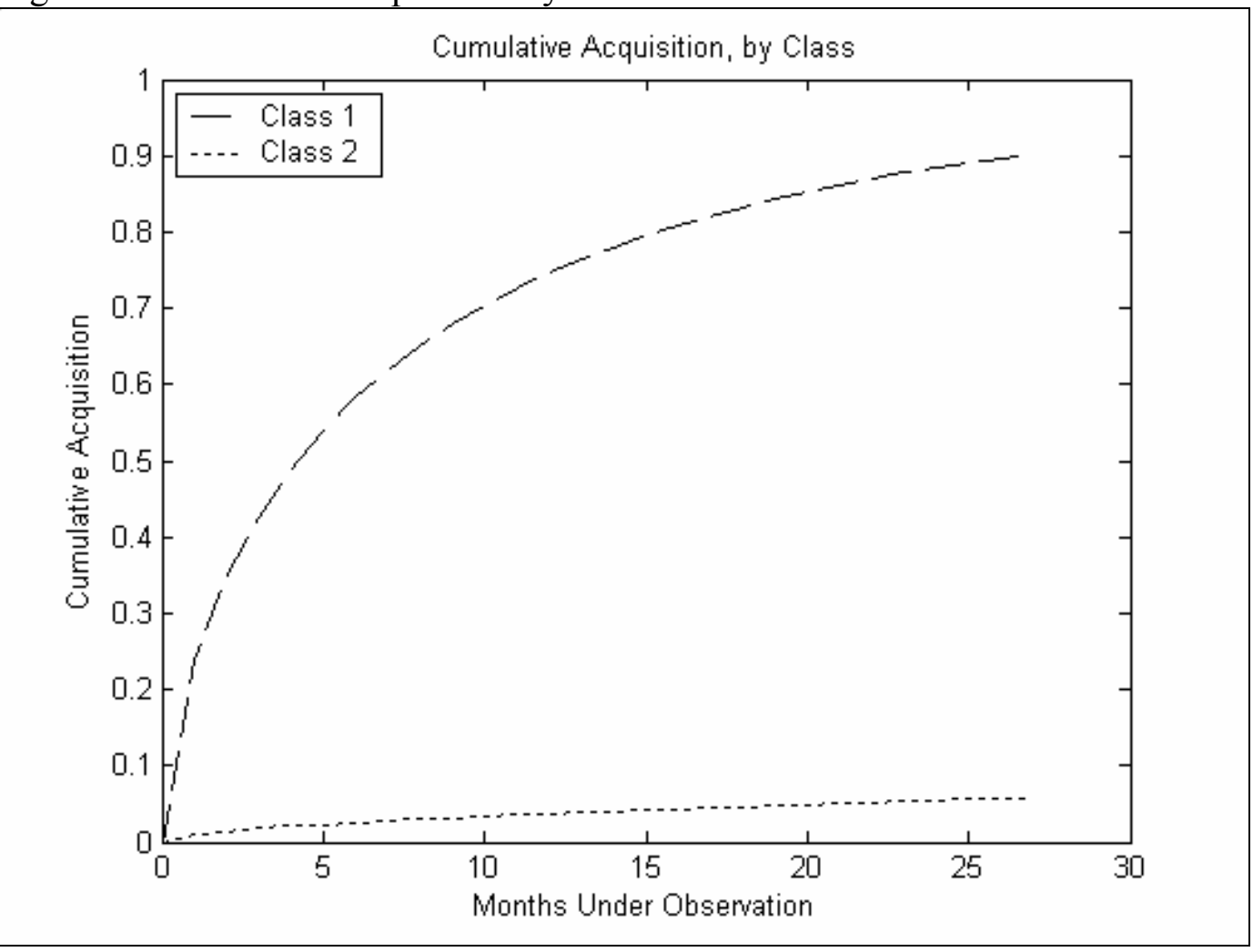

Figure 3b. Service Retention by latent class




Figure 4a. Cumulative Acquisition



Figure 4b. Service Retention



\footnotetext{
* The kink at the end of the actual retention curve results because the few subscribers who came under observation in February 2002 and started service after one month discontinued service by the end of the observation period.
} 
Figure 5. Discounted Expected Tenure






\section{References}

Berger, Paul D. and Nada Nasr-Bechwati (2001), "The Allocation of Promotion Budget to Maximize Customer Equity," Omega, 29 (1), 49-61.

Bitran, Gabriel R. and Susana V. Mondschein (1996), "Mailing Decisions in the Catalog Sales Industry," Management Science, 42 (9), 1364-1381.

Blattberg, Robert C. and John Deighton (1996), "Manage Marketing by the Customer Equity Test," Harvard Business Review, July, 136-144.

Danaher, Peter J. (2006), "Modeling Page Views Across Multiple Websites with an Application to Internet Reach and Frequency Prediction," Marketing Science, forthcoming.

Danaher, Peter J. and Bruce G.S. Hardie (2005), "Bacon with Your Eggs? Applications of a New Bivariate Beta-Binomial Distribution," The American Statistician, 59 (4), 282-286.

Fader, Peter S. and Bruce G.S. Hardie (2006), "How to Project Customer Retention," working paper.

Fader, Peter S., Bruce G.S. Hardie, and Ka Lok Lee (2005), "RFM and CLV: Using Iso-value Curves for Customer Base Analysis," Journal of Marketing Research, 42 (4), 415-430.

Farlie, D.J.G. (1960), "The performance of some correlation coefficients for a general bivariate distribution," Biometrika, 47(December), 307-323.

Gupta, Sunil, Donald R. Lehmann and Jennifer Ames Stuart (2004), "Valuing Customers," Journal of Marketing Research, 41 (1), 7-18.

Hansotia, Behram J. and Paul Wang (1997), "Analytical Challenges in Customer Acquisition," Journal of Direct Marketing, 11 (2), 7-19.

Jain, Dipak and Siddharth Singh (2002), "Customer Lifetime Value Research in Marketing: A Review and Future Directions," Journal of Interactive Marketing, 16 (2), 34-46.

Kamakura, Wagner and Gary Russell (1989), "A Probabilisitic Choice Model for Market Segmentation and Elasticity Structure," Journal of Marketing Research, 26 (November), 379390.

Johnson, Norman L. and Samuel Kotz (1975), “On some generalized Farlie-GumbelMorgenstern distributions," Communications in Statistics, 4(5), 415-427.

Johnson, Norman L. and Samuel Kotz (1977), "On some generalized Farlie-GumbelMorgenstern distributions-II: Regression, correlation, and further generalizations," Communications in Statistics: Theory and Methods, A6(6), 485-496. 
Lee, Mei-Ling Ting (1996), "Properties and Applications of the Sarmanov Family of Bivariate Distributions," Communications in Statistics: Theory and Methods, 25 (6), 1207-1222.

Milne, George R. and Andrew Rohm (2000), "Consumer Privacy and Name Removal Across Direct Marketing Channels: Exploring Opt-in and Opt-out Alternatives," Journal of Public Policy and Marketing, 19 (2), 238-249.

Morrison, Donald G. and David C. Schmittlein (1980), "Jobs, Strikes, and Wars: Probability Models for Duration," Organizational Behavior and Human Performance, 25 (2), 224-251.

Park, Young-Hoon and Peter S. Fader (2004), "Modeling Browsing Behavior at Multiple Websites," Marketing Science, 23 (Summer), 280-303.

Reinartz, Werner and V. Kumar (2002), “The Mismanagement of Customer Loyalty," Harvard Business Review, 80 (July), 86-94.

Reinartz, Werner, Jacquelyn S. Thomas and V. Kumar (2005), "Balancing Acquisition and Retention Resources to Maximize Customer Profitability," Journal of Marketing, 69 (January), 63-79.

Thomas, Jacquelyn S. (2001), "A Methodology for Linking Customer Acquisition and Customer Retention," Journal of Marketing Research, 38 (2), 262-268. 